\title{
Acute Cerebral Stroke with Multiple Infarctions and COVID-19, France, 2020
}

\section{Souheil Zayet, Timothée Klopfenstein, Róbert Kovàcs, Silviu Stancescu, Beate Hagenkötter}

Author affiliation: Nord Franche-Comté Hospital, Trévenans, France

DOI: https://doi.org/10.3201/eid2609.201791

We describe 2 cases in coronavirus disease patients in France involving presumed thrombotic stroke that occurred during ongoing anticoagulation treatment for atrial fibrillation stroke prophylaxis; 1 patient had positive antiphospholipid antibodies. These cases highlight the severe and unique consequences of coronavirus diseaseassociated stroke.

Coronavirus disease (COVID-19) is an infectious disease caused by severe acute respiratory syndrome coronavirus 2 (SARS-CoV-2). Evidence increasingly shows that SARS-CoV-2 is not always confined to the respiratory tract but can induce neurologic diseases (1). Several studies have reported that acute ischemic stroke can develop in COVID-19 patients (1-6). We describe 2 COVID-19 patients who had multiple cerebral infarctions; 1 patient had positive antiphospholipid antibodies.

On March 25, 2020, an 84-year-old man with a history of diabetes mellitus, arterial hypertension, coronary heart disease, peripheral arterial disease, and atrial fibrillation (treated with apixaban [2.5 $\mathrm{mg}$ orally $2 \times / d$ ]) sought care for respiratory symptoms, including dyspnea and cough. At admission, physical examination revealed a blood pressure of $120 / 70 \mathrm{~mm}$ $\mathrm{Hg}$, irregular heartbeat (100 beats/min), fever $\left(39^{\circ} \mathrm{C}\right)$, and bilateral crackling sounds on pulmonary auscultation. Laboratory findings revealed low leukocyte count and lymphopenia (Appendix, https://wwwnc.cdc.gov/EID/article/26/9/20-1791-App1.pdf). Chest radiograph showed a bilateral interstitial infiltrate. Real-time reverse transcription PCR on a nasopharyngeal swab specimen confirmed COVID-19.

Supportive treatment began (oxygen support, antimicrobial drugs [ceftriaxone $1 \mathrm{~g}$ by intravenous (IV) infusion/d], and hydroxychloroquine [200 mg orally $2 \times / d]$ ), and the same dosage of apixaban was continued. On April 3 (day 9 of hospitalization), dysarthria, left hemiplegia, and alteration of consciousness developed. Brain magnetic resonance imaging revealed acute ischemic stroke in multiple vascular areas
(Figure). We switched the anticoagulation medication from apixaban to IV unfractionated heparin [18 $\mathrm{UI} / \mathrm{kg} / \mathrm{h}]$ ). On April 6 (day 12), the patients Glasgow coma scale score was $3 / 15$ (eye opening $=1$, motor response $=1$, verbal response $=1)$, and severe acute respiratory distress developed. No neurologic recovery occurred, and the patient did not undergo subsequent brain imaging. Mechanical ventilation was not possible (high Charlson comorbidity index), and the patient died on April 12 (day 18).

On April 3, a 74-year-old man with a history of multiple cardiovascular diseases, such as atrial fibrillation treated with rivaroxaban $(20 \mathrm{mg}$ orally $1 \times / \mathrm{d})$, sought care for influenza-like illness and confusion. His work colleagues had noticed disorientation during his activity as a truck driver. At admission, physical examination revealed hypertension $(230 / 70 \mathrm{~mm}$ $\mathrm{Hg}$ ) and irregular heartbeat (86 beats/min). He was febrile $\left(38.3^{\circ} \mathrm{C}\right)$ and had crackling sounds on pulmonary auscultation. Neurologic examination showed nonfluent aphasia. COVID-19 was diagnosed from results of real-time reverse transcription PCR, microbiologic testing, and computed tomographic thoracic imaging (Appendix). Brain computed tomographic scan revealed many recent ischemic infarctions in different vascular areas, and magnetic resonance imaging of the brain confirmed this finding (Figure). As with patient 1, this patient had no non-central nervous system thrombotic events (e.g., pulmonary embolisms, abdominal visceral infarction). Treatment began with IV unfractionated heparin $(18 \mathrm{UI} / \mathrm{kg} / \mathrm{h})$, hydroxychloroquine $(200 \mathrm{mg}$ orally $2 \times / d$ ), and antimicrobial drugs (ceftriaxone $1 \mathrm{~g}$ by IV infusion/d). The patient's aphasia regressed, and he was discharged on April 20.

Several factors can cause acute ischemic stroke, but the primary ones are arterial and cardiac embolism, arterial wall disease, and variants of those conditions. Both of these patients had concurrent cardiovascular conditions, particularly atrial fibrillation, although both were adequately treated with anticoagulants. Hematologic derangements, including lymphopenia and leukopenia, are associated with ischemic stroke and are predictors of worse prognosis with stroke (7). A systematic review and meta-analysis identified lymphopenia as one of the most prevalent laboratory results described in COVID-19 (35\%-72\%) (8), and we observed it in these 2 patients. Many infectious agents have been implicated as potential causes of cerebral stroke, such as herpes simplex virus, varicella zoster virus, Treponema pallidum, Mycobacterium tuberculosis, and Aspergillus spp.; acute bacterial meningitis has also been 

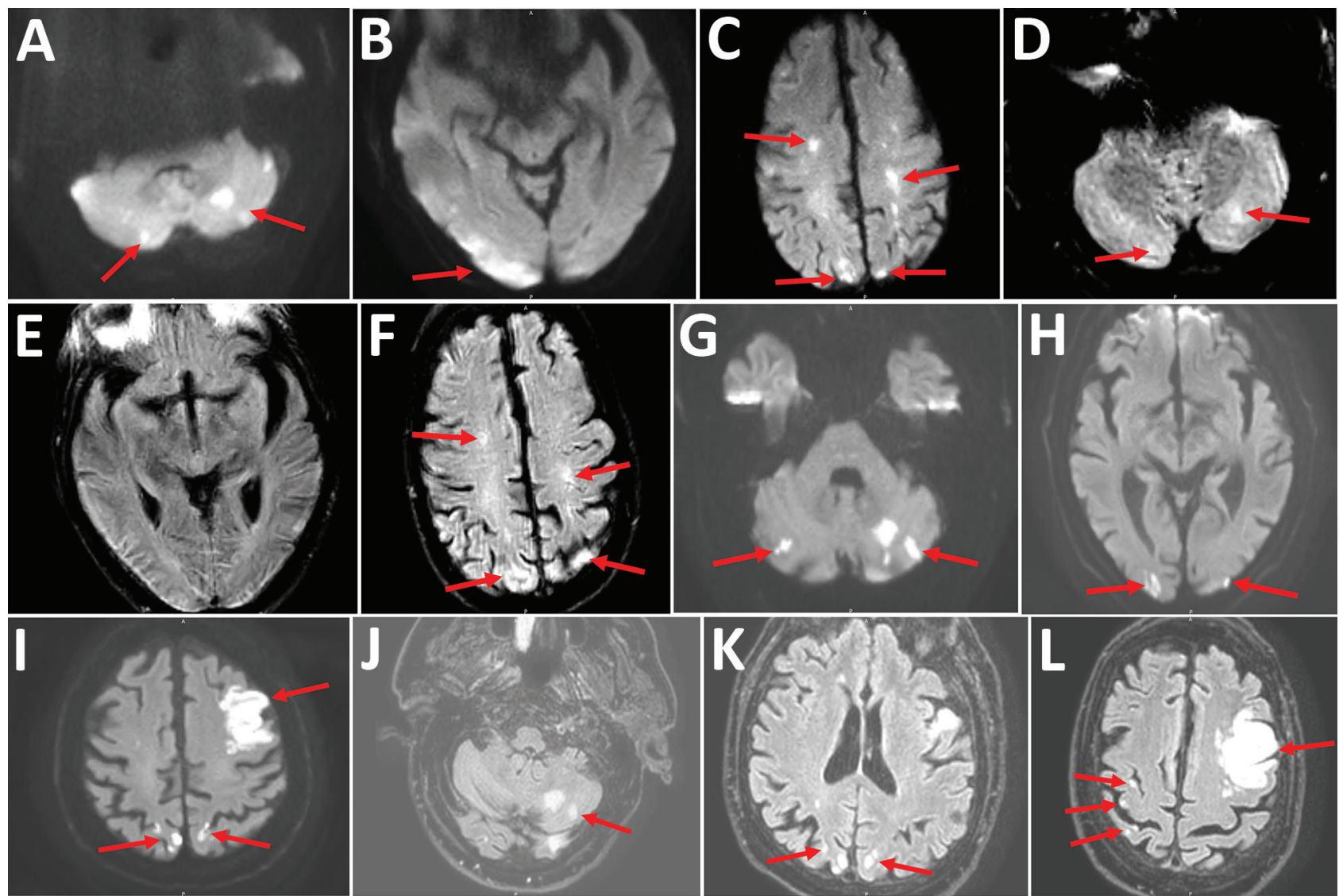

Figure. Cerebral magnetic resonance image (MRI) showing acute ischemic stroke in multiple vascular areas of 2 coronavirus disease patients, France. A-F) Patient 1. Diffusion weighted imaging (DWI) showed hyperintensive lesions of bilateral cerebellar hemispheres (arrows, A), right occipital cortex (arrows, B), bilateral centrum semiovale and bilateral parietal cortex (arrows, C). A part of the lesions are already hyperintensive in FLAIR (fluid-attenuated inversion recovery) sequences (arrows, D, F). Normal FLAIR sequence of the right occipital cortex; early stroke MRI (E). MRI quality is reduced because of dental artifact. G-L) Patient 2. Cerebral MRI showed multiple small ischemic infarctions with hyperintensive lesions (arrows) in bilateral cerebellar hemispheres (DWI [G], FLAIR [J; only left hemisphere]), bilateral occipital cortex (DWI [H], FLAIR [K]), main infarction in the left frontal lobe and small biparietal infarctions (DWI [I], FLAIR [L]).

implicated (9). Multiple brain localizations have previously been described with other viruses that lead to cerebrovascular complications through various mechanisms, including multifocal vasculopathy, focal infiltrative vasculitis and vasospasm, and direct vessel wall invasion and thrombus formation (10).

In this rapidly emerging epidemic, several cases have reported strokes in SARS-CoV-2-infected patients $(1-6)$. However, the unique feature in the patients we report is multiple simultaneous strokes. These cases involved presumed thrombotic stroke that occurred during ongoing anticoagulation for atrial fibrillation stroke prophylaxis. Given the increasing realization that COVID-19 might be associated with hypercoagulability, the concurrent presence of anticoagulation with direct oral anticoagulants should not be reassuring as preventive.

Other authors suggest that the presence of antiphospholipid antibodies, such as anticardiolipin antibodies, as well as anti- $\beta_{2}$-glycoprotein I antibodies might rarely lead to multiple thrombotic cerebral events (5). In the patients we report, subsequent serologic testing showed anticardiolipin antibodies (IgM) in patient 1 . We did not conduct functional testing (such as dilute Russell viper venom time). However, antiphospholipid antibody syndrome in cases of stroke cannot be diagnosed until positive antibodies persist after multiple months.

The association between cerebral stroke and COVID-19 requires more attention. Coagulability dysfunction and possibly antiphospholipid antibody syndrome may contribute to thromboembolic events in the central nervous system. Further investigation is required to determine the prognostic role of the presence of antiphospholipid antibodies in COVID-19.

\section{Acknowledgments}

We thank Vincent Gendrin for English language editing and his support and valuable feedback. 


\section{About the Author}

Dr Zayet is a specialist in the Infectious Diseases Department of Nord Franche-Comte Hospital, France. His primary research interests focus on hepatitis and tuberculosis, especially in HIV-infected patients.

\section{References}

1. Mao L, Jin H, Wang M, Hu Y, Chen S, He Q, et al. Neurologic manifestations of hospitalized patients with coronavirus disease 2019 in Wuhan, China. JAMA Neurol. 2020 Apr 10 [Epub ahead of print]. https://doi.org/10.1001/ jamaneurol.2020.1127

2. Oxley TJ, Mocco J, Majidi S, Kellner CP, Shoirah H, Singh IP, et al. Large-vessel stroke as a presenting feature of covid-19 in the young. N Engl J Med. 2020;382:e60. https:/ / doi.org/ 10.1056/NEJMc2009787

3. Klok FA, Kruip MJHA, van der Meer NJM, Arbous MS, Gommers DAMPJ, Kant KM, et al. Incidence of thrombotic complications in critically ill ICU patients with COVID-19. Thromb Res. 2020 Apr 10 [Epub ahead of print]. https:/ / doi.org/10.1016/j.thromres.2020.04.013

4. Lodigiani C, Iapichino G, Carenzo L, Cecconi M, Ferrazzi P, Sebastian T, et al.; Humanitas COVID-19 Task Force. Venous and arterial thromboembolic complications in COVID-19 patients admitted to an academic hospital in Milan, Italy. Thromb Res. 2020;191:9-14. https://doi.org/10.1016/ j.thromres.2020.04.024

5. Zhang Y, Xiao M, Zhang S, Xia P, Cao W, Jiang W, et al. Coagulopathy and antiphospholipid antibodies in patients with covid-19. N Engl J Med. 2020;382:e38. https:/ / doi.org/ 10.1056/NEJMc2007575

6. Griffin DO, Jensen A, Khan M, Chin J, Chin K, Parnell R, et al. Arterial thromboembolic complications in COVID-19 in low risk patients despite prophylaxis. Br J Haematol. 2020 May 6 [Epub ahead of print]. https://doi.org/10.1111/ bjh.16792

7. Ren H, Liu X, Wang L, Gao Y. Lymphocyte-to-monocyte ratio: a novel predictor of the prognosis of acute ischemic stroke. J Stroke Cerebrovasc Dis. 2017;26:2595-602. https:/ / doi.org/10.1016/j.jstrokecerebrovasdis.2017.06.019

8. Rodriguez-Morales AJ, Cardona-Ospina JA, Gutiérrez-Ocampo E, Villamizar-Peña R, Holguin-Rivera Y, Escalera-Antezana JP, et al.; Latin American Network of Coronavirus Disease 2019-COVID-19 Research (LANCOVID-19). Electronic address: https://www. lancovid.org. Clinical, laboratory and imaging features of COVID-19: A systematic review and meta-analysis. Travel Med Infect Dis. 2020;34:101623. https://doi.org/10.1016/ j.tmaid.2020.101623

9. Shulman JG, Cervantes-Arslanian AM. Infectious etiologies of stroke. Semin Neurol. 2019;39:482-94. https://doi.org/10.1055/s-0039-1687915

10. Lin C-F, Hong C-T, Lee W-H, Wu D, Hu C-J, Chung C-C. Disseminated cutaneous herpes zoster and multiple cerebral infarcts in an adult with diabetes mellitus. J Neurovirol. 2020;26:130-2. https:// doi.org/10.1007/ s13365-019-00790-7

Address for correspondence: Souheil Zayet, Department of Infectious Disease, Nord Franche-Comte Hospital, 100 Route de Moval, 90400 Trevenans, France; email: souhail.zayet@gmail.com

\section{Large SARS-CoV-2 Outbreak Caused by Asymptomatic Traveler, China}

\author{
Jingtao Liu, Jiaquan Huang, Dandan Xiang
}

Author affiliations: Hubei University of Medicine, Shiyan, China (J. Liu); Huazhong University of Science and Technology, Wuhan, China (J. Liu, J. Huang, D. Xiang); Tongji Hospital, Wuhan (J. Huang, D. Xiang)

\section{DOI: https://doi.org/10.3201/eid2609.201798}

An asymptomatic person infected with severe acute respiratory syndrome coronavirus 2 returned to Heilongjiang Province, China, after international travel. The traveler's neighbor became infected and generated a cluster of $\geq 71$ cases, including cases in 2 hospitals. Genome sequences of the virus were distinct from viral genomes previously circulating in China.

C oronavirus disease (COVID-19), caused by severe acute respiratory syndrome coronavirus 2 (SARS-CoV-2), has spread rapidly around the world since the first cases were reported in late $2019(1,2)$. Prior to April 9, 2020, Heilongjiang Province, China, had not reported a new COVID-19 diagnosis since March 11, 2020. On April 9, SARS-CoV-2 was diagnosed in 4 patients. By April 22, $\geq 71$ persons had been infected. The likely origin of this cluster is an imported case from an asymptomatic traveler.

We collected and analyzed epidemiologic data published on the website of the Health Commission of Heilongjiang Province for April 9-23, 2020 (3). We defined confirmed COVID-19 cases as persons who tested positive for SARS-CoV-2 and had clinical symptoms. We defined asymptomatic carriers as persons without clinical symptoms who tested positive for SARS-CoV-2. We refer to case-patients by a letter for each family (A-Z, AA-ZZ), then by the assumed transmission generation (1-2), and finally in sequential order of exposure to SARS-CoV-2-positive persons in generations 1-3 (Figure) (4).

On March 19, 2020, case-patient A0 returned to Heilongjiang Province from the United States; she was asked to quarantine at home. She lived alone during her stay in Heilongjiang Province. She had negative SARS-CoV-2 nucleic acid and serum antibody tests on March 31 and April 3.

Patient B1.1 was the downstairs neighbor of casepatient A0. They used the same elevator in the building but not at the same time and did not have close contact otherwise. On March 26, B1.1's mother, B2.2, and her mother's boyfriend, B2.3, visited and stayed in B1.1's 